\title{
Combined Inversion of Early Arrival P-wave and Rayleigh Wave with Cross-gradient Constraint
}

\author{
Lei Fu, Sixin Liu \\ College of Geo-exploration Science and Technology \\ Jilin University \\ Changchun, China \\ liusixin@jlu.edu.cn
}

\begin{abstract}
We present a combined early arrival P-wave and Rayleigh wave inversion strategy that uses the cross-gradient between the $\mathbf{P}$-velocity and S-velocity as a regularization term. First, the P-velocity model is inverted by the early arrival full wave inversion (FWI) method. Then the magnitude spectra of the surface waves in the frequency-wavenumber domain are inverted for the S-velocity model with a cross-gradient constraint. This constraint regularization insists that the S-velocity gradient is closely parallel to that of the P-velocity gradient. Results show that cross-gradient regularization provides a significant reduction in artifacts in the S-velocity tomogram.
\end{abstract}

\section{Keywords—combined inversion; rayleigh wave}

\section{INTRODUCTION}

In seismic exploration, knowledge of the near-surface velocity can improve the construction of an accurate geological model. Until recently, most surface-wave inversion approaches assumed a layered model, which is not justified in a complex geological environment. To overcome this 1D limitation, researchers have begun to explore the benefits of 2D waveform inversion of surface waves. WindowedAmplitude waveform inversion as a replacement to classical FWI, which has faster convergence, however, lower spatial resolution in the tomogram is deduced. To partly recover this lost resolution we propose a combined inversion of early arrival P-wave data and Rayleigh wave data. In this case the less robust inversion of surface waves is combined with high resolution $\mathrm{P}$-wave tomography to achieve better spatial resolution of the $\mathrm{S}$-wave velocity tomogram.

Combinedcombined inversion for different gophsical data sets data can be classified into two categories. The first category is based on the petrophysical relationship between different parameters. The second category of combined inversion utilizes the structural similarity between different parameter profiles. As an example, Gallardo and $\left.\mathrm{Meju}^{[1}\right]$ successfully used the cross-gradient approach for joint inversion of DC resistivity and seismic traveltime data. It is this second category of combined inversion that we will employ in this work.

\section{THEORY}

\section{A. Cross-Gradient Definition}

The definition of the cross-gradient is expressed as the cross product of two individual model gradients:

$$
\mathbf{t}\left(m^{p}, m^{s}\right)=\nabla m^{p}(x, y, z) \times \nabla m^{s}(x, y, z),
$$

where $m^{p}$ is the P-wave velocity model, and $m^{s}$ is the Svelocity model. The cross-gradient regularization term is based on the fact that the structural similarity of two modelparameters reaches its maximum when the cross-gradient achieves its minimum.

\section{B. Early Arrival P-wave FWI}

The FWI method is designed to minimize the following objective function:

$$
\Phi^{p}\left(\mathbf{v}^{p}\right)=\frac{1}{2}\left\|\Delta \mathrm{d}_{r s}\right\|^{2},
$$

where $\Delta \mathrm{d}_{r s}$ is the data residual between the predicted and observed data. The P-velocity distribution can be iteratively updated using any gradient based method such as the conjugate gradient method:

$$
\mathbf{v}_{k+1}^{p}=\mathbf{v}_{k}^{p}+\alpha_{k}^{p} \mathbf{d}_{k}^{p}
$$

where $\mathbf{v}_{k}^{p}$ is the P-velocity model at the $\mathrm{k}^{\text {th }}$ iteration; $\alpha_{k}^{p}$ is the step length, which can be found by a line-search algorithm; and $\mathbf{d}_{k}^{p}$ is the update direction:

$$
\begin{gathered}
\mathbf{d}_{k}^{p}=-\mathbf{g}_{k}^{p}+\beta_{k}^{p} \mathbf{d}_{k-1}^{p}, \\
\beta_{k}^{p}=\frac{\left(\mathbf{g}_{k}^{p}-\mathbf{g}_{k-1}^{p}, \mathbf{g}_{k}^{p}\right)}{\left(\mathbf{g}_{k-1}^{p}, \mathbf{g}_{k-1}^{p}\right)} .
\end{gathered}
$$

The current search direction $\mathbf{d}_{k}^{p}$ is given by the derivative of the misfit function with respect to the current model parameter $\mathbf{g}_{k}^{p}$ and the former search direction $\mathbf{d}_{k-1}^{p}$. The Gradient $\mathbf{g}_{k}^{p}$ is given by

$$
\mathbf{g}^{p}=\frac{1}{\left(v^{p}\right)^{3}} \sum_{s} \int \dot{p}_{c a l}\left(x, t \mid x_{s}\right) \dot{p}^{\prime}\left(x, t \mid x_{r}\right) d t,
$$

where 


$$
\dot{p}^{\prime}\left(x, t \mid x_{s}\right)=\sum_{r} G^{p}\left(x,-t \mid x_{r}, 0\right) * \Delta \mathrm{d}\left(x_{r}, t \mid x_{s}\right),
$$

and the symbol * represents temporal convolution, $\dot{p}\left(x, t \mid x_{s}\right)$ represents the time derivative of $p\left(x, t \mid x_{s}\right)$, and $G^{p}$ is the Green's function associated with constant density equation for the velocity field $v^{p}$.

\section{Amplitude Waveform Inversion for Rayleigh Wave}

The amplitude waveform inversion ${ }^{[2]}$ is an alternative to FWI, which focuses on minimizing the difference of the absolute spectrum value of the predicted and observed seismograms:

$$
\Phi^{s}\left(\mathbf{v}^{s}, \mathbf{v}^{p}\right)=\frac{1}{2} \sum_{w}\left\|D_{w}^{c a l}|-| D_{w}^{o b s} \mid\right\|^{2},
$$

where $\left|D_{w}^{c a l}\right|$ and $\left|D_{w}^{o b s}\right|$ are the amplitude spectra of the calculated and observed data in the F-K domain. Similar to updating the $\mathrm{P}$ velocity, the $\mathrm{S}$ velocity can be updated iteratively by:

$$
\mathbf{v}_{k+1}^{s}=\mathbf{v}_{k}^{s}+\alpha_{k}^{s} \mathbf{d}_{k}^{s}
$$

where $\mathbf{v}_{k}^{s}$ is the S-velocity model at the $\mathrm{k}^{\text {th }}$ iteration; $\mathbf{d}_{k}^{s}$ is the search direction and $\alpha_{k}^{s}$ is the step length. The search direction is given by the former search direction and the current gradient:

$$
\mathbf{g}^{s}=\frac{1}{\left(\mathbf{v}^{s}\right)^{3}} \sum_{s} \int \dot{u}_{z}\left(x, t \mid x_{s}\right)_{c a l} \dot{u}_{z}^{\prime}\left(x, t \mid x_{r}\right) d t,
$$

where

$$
\dot{u}_{z}^{\prime}\left(x, t \mid x_{s}\right)=\sum_{r} G^{s}\left(x,-t \mid x_{r}, 0\right) * \delta d\left(x_{r}, t \mid x_{s}\right),
$$

and $\dot{u}_{z}\left(x, t \mid x_{s}\right)$ is the vertical displacement component calculated using the current S-velocity model, and is $G^{s}$ the Green's function associated with elastic wave equation for the S-velocity $v^{s}$. The term $\dot{u}_{z}^{\prime}\left(x, t \mid x_{r}\right)$ is the vertical component of the wavefield calculated by back propagating the pseudoresidual $\delta d\left(x_{r}, t \mid x_{s}\right)$ in reverse time, and the pseudo-residual

$$
\delta d\left(x_{r}, t \mid x_{s}\right)=\sum_{w} w_{w}^{T} \mathfrak{R}\left\{F_{2 D}^{-1}\left\{\frac{D_{w}^{c a l}}{\left|D_{w}^{c a l}\right|}\left(\left|D_{w}^{c a l}\right|-\left|D_{w}^{o b s}\right|\right)\right\}\right\},
$$

\section{Combined Inversion Objective Function}

To improve the accuracy of the inversion results, we jointly invert for the P-velocity or the S-velocity tomograms with a cross-gradient constraint. The combined inversion objective function with cross-gradient regularization for early arrival P-wave FWI is

$$
\Phi^{p}\left(\mathbf{v}^{p}, \mathbf{v}^{s}\right)=\underbrace{\frac{1}{2}\left\|\Delta d_{r s}\right\|^{2}}_{\Phi_{0}^{p}}+\underbrace{\frac{\lambda_{1}}{2}\left\|t\left(\mathbf{v}^{p}, \mathbf{v}^{s}\right)\right\|^{2}}_{\psi^{p}},
$$

and the combined objective function with cross-gradient regularization for the amplitude inversion of Rayleigh waves is

$$
\Phi^{s}\left(\mathbf{v}^{s}, \mathbf{v}^{p}\right)=\underbrace{\frac{1}{2} \sum_{w}\left\|D_{w}^{c a l}|-| D_{w}^{o b s} \mid\right\|^{2}}_{\Phi_{0}^{s}}+\underbrace{\frac{\lambda_{2}}{2}\left\|t\left(\mathbf{v}^{s}, \mathbf{v}^{p}\right)\right\|^{2}}_{\psi^{s}},
$$

where $\lambda_{1}$ and $\lambda_{2}$ are regularization parameters. If the regularization parameters $\lambda_{1}$ and $\lambda_{2}$ are set to be zero at the same time, equations 13 and 14 degrade to uncoupled inversion schemes.

\section{NUMERICAL EXAMPLES}

The combined inversion procedure is tested on data generated for the P- and S-velocity models shown in Figure 1. In this example, the P- and S-velocity models obey a linear relationship and the model is discretized into 30x100 gridpoints with the grid size of $3 \mathrm{~m}$. The red $\mathrm{X}$ in Figure 1 indicates the source location with a $15 \mathrm{~m}$ source interval, and there are 100 receivers spaced $3 \mathrm{~m}$ apart. The source wavelet is a Ricker wavelet with the peak frequency of $30 \mathrm{~Hz}$.
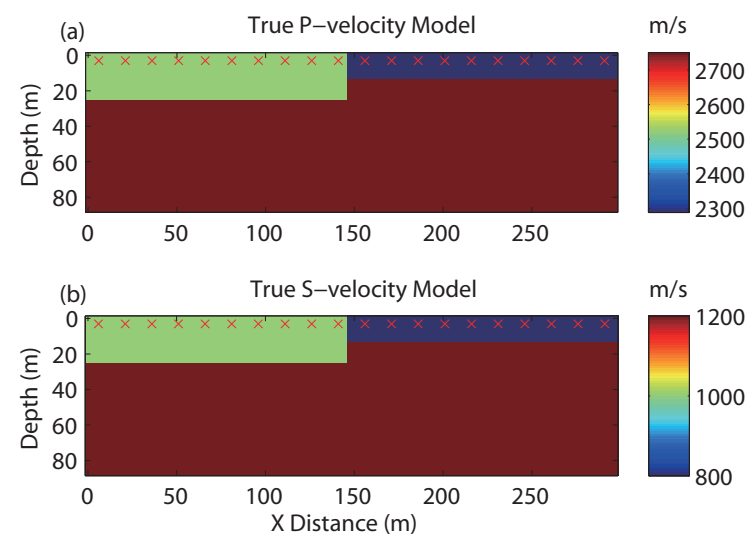

Fig.1 (a) The true P-velocity and (b) the S-velocity models, where the red X's indicate the source locations.
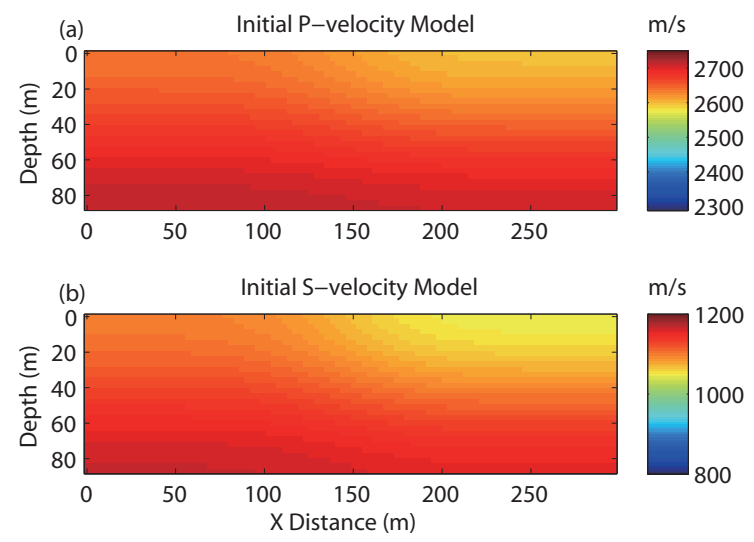

Fig.2 Initial (a) P-velocity and (b) S-velocity models. 

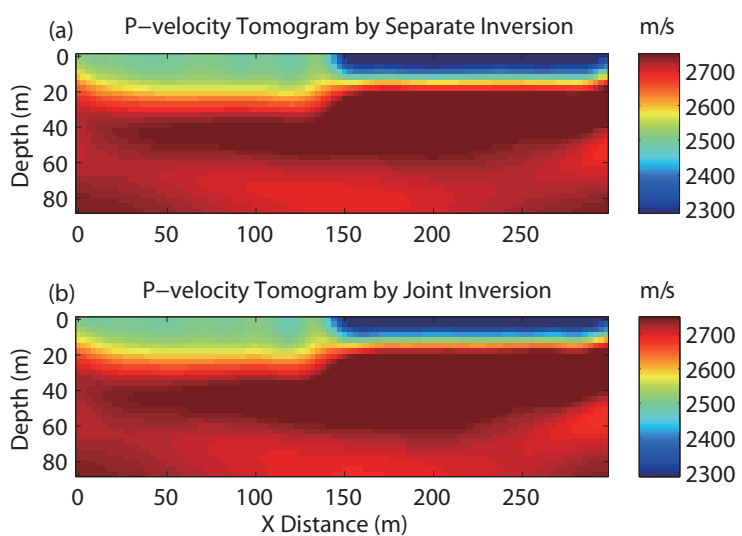

Fig.3 Tomograms obtained by the early arrival FWI method applied to early arrival P waves. (a) Separate inversion P-velocity tomogram at the 10th iteration, (b) combined inversion P-velocity tomogram at the 10th iteration
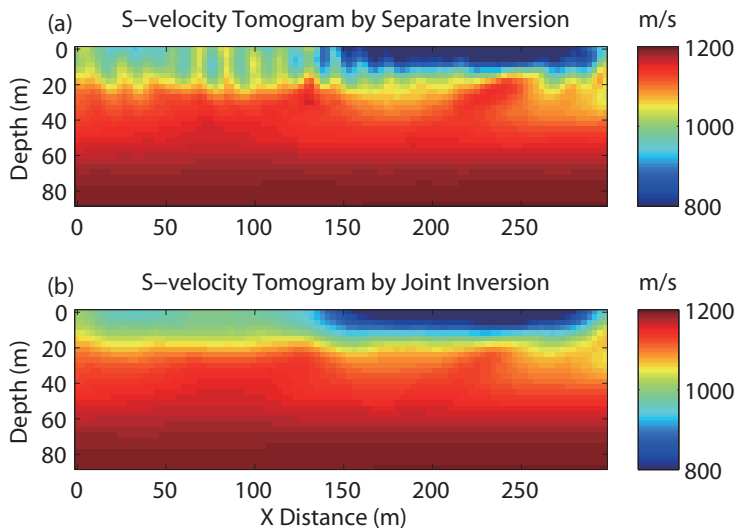

Fig.4 Inversion results for Rayleigh waves. (a) Separate inversion S-velocity tomogram at the 10th iteration, (b) combined inversion S-velocity tomogram at the 10 th iteration.

The initial-velocity models are shown in Fig.2. Fig.3(a) is the separate inversion tomogram after 10 iterations of early arrival $\mathrm{P}$ waves and Fig.3(b) is the combined inversion tomogram for $\mathrm{P}$ waves after 10 iterations inversion. We can hardly see any improvement for the P-velocity tomogram, because the inverted $\mathrm{P}$ velocity using the separate inversion method is quite accurate already. Further improvement by combined inversion method is unlikely. For the S-velocity tomograms shown in Fig.4, some artifacts appear in the nearsurface region (0-20 $\mathrm{m}$ in depth) for the separate inversion result in Fig.4(a). However, the artifacts are eliminated and the interface between layers is reinforced in the combined inversion tomogram in Fig.4(b).

\section{FIELD DATA}

A 2D seismic survey was carried out to detect an unknown buried fault. The survey consists of 240 shots and 240 vertical-component geophones for each shot, with a uniform spacing interval of $5 \mathrm{~m}$ for both shots and receivers. The recording time is $3 \mathrm{~s}$ and the sampling rate is $1 \mathrm{~ms}$. There is a fault located around shot \#55 according to the common offset gathers and we use shot gathers from \#21 to \#81 in our inversion.

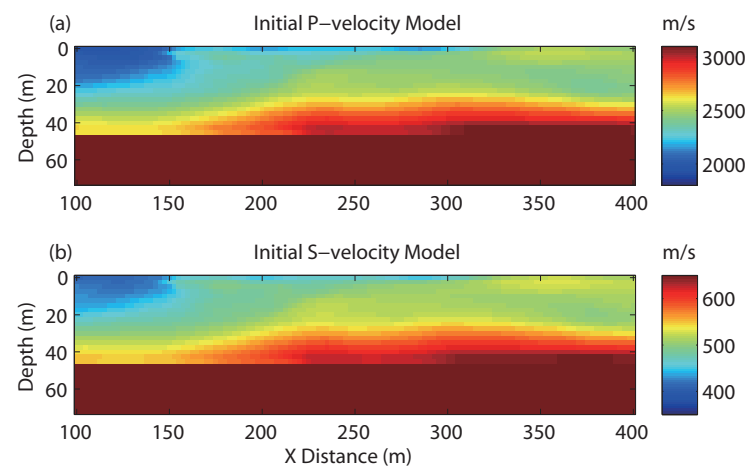

Fig.5 Initial-velocity models. (a) Initial P-velocity model estimated based on the S-velocity model shown in (b), (b) initial S-velocity model computed from $1 \mathrm{D}$ surface-wave dispersion inversion

An initial S-velocity model is computed by $1 \mathrm{D}$ surfacewave dispersion inversion and is shown in Fig.5(b). The initial S-velocity model contains 30x121 grid-points with a spacing interval of $2.5 \mathrm{~m}$. Within the depth of $30 \mathrm{~m}$, the S velocity increases from $420 \mathrm{~m} / \mathrm{s}$ to $530 \mathrm{~m} / \mathrm{s}$ laterally, the low-velocity anomaly begins at the offset of $100 \mathrm{~m}$ and ends at about $150 \mathrm{~m}$ in the X-direction. This suggests that the fault location is possibly located between 150 and $170 \mathrm{~m}$; the S velocity beyond $30 \mathrm{~m}$ in depth is a high velocity area, so we enforce a model velocity of $650 \mathrm{~m} / \mathrm{s}$ below the depth of $45 \mathrm{~m}$. Based on the initial S-velocity model, an initial P-velocity model (Figure 5(a)) is estimated.

The separate inversion result for $\mathrm{P}$ waves after 15 iterations is shown in Fig.6(a), and the combined inversion result after 15 iterations is shown in Fig.6(b). We cannot get much improvement from the combined inversion for the Pvelocity tomogram. Compared with the initial velocity model, we find that the low-velocity anomaly area becomes larger, and the possible fault locations are at $230 \mathrm{~m}<\mathrm{x}<280 \mathrm{~m}$. The inversion result is more consistent with the common offset gather results.

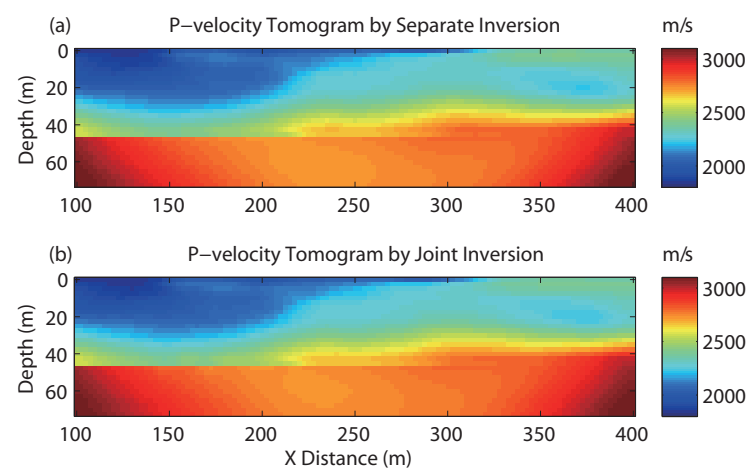

Fig.6 Inversion results for early-arrival $\mathrm{P}$ waves. (a) Separate inversion Pvelocity tomogram at the $15^{\text {th }}$ iteration, (b) combined inversion P-velocity tomogram at the $15^{\text {th }}$ iteration.

The separate inversion result for Rayleigh waves is shown in Fig. 7(a), where there still is a low-velocity anomaly from $100 \mathrm{~m}$ to $200 \mathrm{~m}$ and the shape of this anomaly is different from that of the P-velocity tomogram shown in Fig.6(a). In addition, a high-velocity anomaly can be found from 250 to 
$300 \mathrm{~m}$ down to a depth of $20 \mathrm{~m}$. As for the combined inversion result shown in Fig.7(b), the shape of the lowvelocity anomaly is quite similar to that in the P-velocity tomogram. Furthermore, the high-velocity anomaly seen in the separate inversion tomogram disappears in the combined inversion. The combined inversion results show better structural similarity between the P-velocity and S-velocity tomograms compared with the separate inversion results.
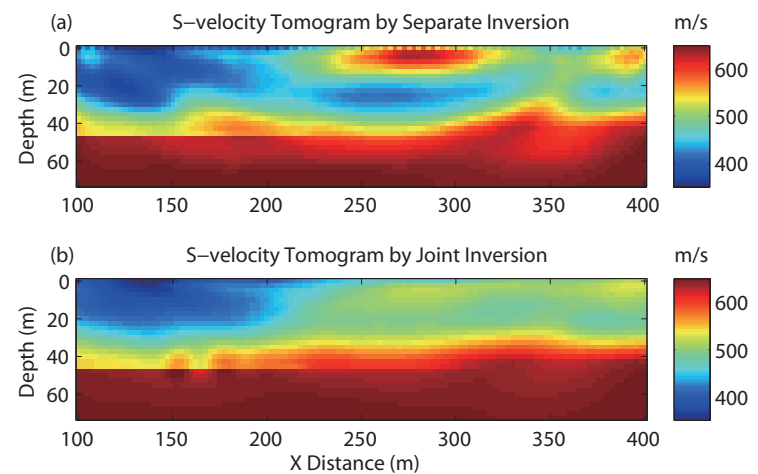

Fig.7 Inversion results for Rayleigh waves. (a) Separate inversion S-velocity tomogram at the $15^{\text {th }}$ iteration, (b) combined inversion S-velocity tomogram at the $15^{\text {th }}$ iteration.

\section{CONCLUSION}

A combined inversion method with a cross-gradient constraint is presented. This method jointly inverts the firstarrival P waves and the Rayleigh waves by FWI and AWI, respectively. The combined inversion with cross-gradient regularization provides a noticeable improvement in the Svelocity tomogram compared with the separate inversion for the S-velocity tomogram.

\section{REFERENCES}

[1] Gallardo, L. A., and M. A. Meju, 2003, Characterization of heterogeneous near-surface materials by joint $2 \mathrm{~d}$ inversion of $\mathrm{dc}$ resistivity and seismic data: Geophysical Research Letters, 30.

[2] Solano, C. P., D. Donno, and H. Chauris, 2014, Alternative waveform inversion for surface wave analysis in 2-d media: Geophysical Journal International, 198, 1359-1372. 